\title{
Coevaluación o Evaluación Compartida en el Contexto Universitario: La Percepción del Alumnado de Primer Curso
}

\section{Co-assessment in Higher Education: The Perception of First- year Students}

\author{
Miguel Ángel Gómez Ruiz * \\ Victoria Quesada Serra \\ Universidad de Cádiz
}

\begin{abstract}
Es imprescindible implicar al alumnado en la evaluación para fomentar su aprendizaje. La modalidad participativa más desconocida es la coevaluación o evaluación compartida. Teniendo en consideración el uso polisémico del concepto coevaluación en español, en este estudio se entiende como aquella evaluación negociada entre docentes y estudiantes donde la responsabilidad es compartida y en la que es imprescindible el diálogo. En la literatura específica se destaca que esta modalidad favorece el aprendizaje autónomo y la autorregulación. Para contrastar con la realidad las consecuencias de la evaluación compartida se ha desarrollado una experiencia que implicó a 192 estudiantes universitarios de primer curso durante tres años académicos. Tras su implantación, se realizó un estudio descriptivo mediante un cuestionario on-line para analizar la potencialidad para el aprendizaje, comprobar el grado de satisfacción del alumnado y conocer los aspectos positivos y negativos que detectaron los estudiantes. En los resultados se puede comprobar la elevada satisfacción del alumnado con la coevaluación. Los participantes consideran que la mayor potencialidad para el aprendizaje proviene de la importancia de la retroalimentación que se ofrece, ya que permite ser más conscientes de los errores y aprender de ellos. Igualmente, otros elementos positivos detectados fueron la colaboración y la comunicación entre docente y estudiantes, así como la formación sobre el proceso de evaluación. Es imprescindible continuar con la implantación e indagación de evaluaciones basadas en el diálogo y en compartir significados en el contexto universitario, que permitan a los estudiantes mejorar sus aprendizajes y a los docentes optimizar y flexibilizar sus estrategias de enseñanza.
\end{abstract}

Palabras Clave: Evaluación educativa, Evaluación de estudiantes, Evaluación formativa, Educación Superior, Relación de docentes y estudiantes, Evaluación alternativa, Coevaluación.

Participative assessment strategies such as self-assessment, peer assessment and coassessment enhance student learning. Co-assessment is the least known participative strategy. Considering the polysemous use of the word co-assessment in the Spanish literature in this study the concept is defined and understood as a teacher-student negotiated assessment where the responsibility of assessment is shared and dialogue is key. Coassessment promotes self-regulated and autonomous learning according to the specific literature. To analyse the consequences of co-assessment a three-year study involving 192 first-year students was implemented. An on-line survey was applied to analyse the learning potentialities of co-assessment, students' satisfaction with the experience and the positive and negative aspects perceived. The results show high student satisfaction. Most participants consider a learning potentiality the importance of the feedback provided when implementing co-assessment since it allows them to find out their mistakes and learn from them. Likewise, other positive aspects mentioned include the collaboration and communication established among teachers and students and an increasing of assessment literacy. The authors of the study believe it is important to continue with the implementation and research of dialogue-based assessment practices and in sharing meanings in Higher Education, which might allow students to enhance their learning and teachers to optimise and increase the adaptability of their teaching and learning strategies.

Keywords: Educational assessment, Student evaluation, Formative evaluation, Higher education, Teacher student relationship, Alternative assessment, Co-assessment.

*Contacto: miguel.gomez@uca.es

issn: 1989-0397

www.rinace.net/riee/

https://revistas.uam.es/riee
Recibido: 26 de julio de 2016

$1^{\text {a }}$ Evaluación: 10 de octubre de 2016

Aceptado: $\quad 10$ de noviembre de 2016 


\section{Introducción}

Durante las presentaciones de las asignaturas universitarias es habitual que el profesorado haga hincapié en los objetivos, los contenidos o las competencias de la materia. No obstante, la mayor expectación de los estudiantes -y, en algunos casos, incluso incertidumbre-, aparecerá cuando llega el momento de tratar el tema de la evaluación. Existe, de hecho, cierto consenso en la literatura en considerar que todo lo concerniente a la evaluación despierta en el alumnado un gran interés, siendo numerosos los autores que proponen aprovechar esta relevancia que se le otorga para poner en práctica una evaluación orientada al aprendizaje en la universidad (Carless, 2007; Fernández March, 2010; Gómez, Rodríguez e Ibarra, 2011, 2013; Rodríguez, Quesada e Ibarra, 2016).

Uno de los requisitos para promover una evaluación que fomente el aprendizaje es la implicación del alumnado en el proceso evaluativo, ya sea en la planificación, ejecución o calificación (Quesada, García y Gómez, 2016). No obstante, la forma más común de involucrar y responsabilizar al alumnado en el proceso de evaluación es a través de las diferentes modalidades participativas, nos referimos a la autoevaluación, la evaluación entre iguales y la coevaluación o evaluación compartida y negociada entre docentes y estudiantes.

Los estudios muestran que de las tres modalidades de evaluación mencionadas, la coevaluación es la práctica menos utilizada, la más desconocida y la que más inseguridad produce entre el profesorado universitario (Álvarez Rojo, Padilla Carmona, Rodríguez Santero, Torres Gordillo y Suárez Ortega, 2011; Rodríguez Gómez, Ibarra Sáiz, Gallego Noche, Gómez Ruiz y Quesada Serra, 2012; Quesada, Rodríguez e Ibarra, 2016). De esta forma, en una investigación reciente en la que participaron 427 docentes universitarios, solo el $8,6 \%$ de los encuestados manifestaron sentirse competentes en el uso de la coevaluación en sus clases, expresando el 73,5\% que nunca o en raras ocasiones utilizan esta evaluación compartida (Quesada, Rodríguez e Ibarra, 2016).

En una línea similar de trabajo, otra investigación (Rodríguez, Ibarra y García, 2013) puso de manifiesto que únicamente el $4,02 \%$ de las referencias en inglés sobre modalidades de evaluación en las bases de datos internacionales ERIC (Education Resources Information Center) y Academic Search Premier se corresponden a la coevaluación, el resto se reparte entre la autoevaluación $(56,9 \%)$ y la evaluación entre iguales (39\%). En ese mismo estudio se revela que en las bases de datos en español esta cifra sube al 10,9\% de las referencias, no debiéndose realmente a un mayor uso de la misma, sino al significado polisémico del concepto coevaluación en nuestro idioma.

En efecto, el término coevaluación ha sido utilizado en nuestro contexto para designar dos realidades participativas distintas, la evaluación por compañeros o entre iguales por un lado y la evaluación compartida, colaborativa, cooperativa o negociada por el otro. Es por ello que se considera imprescindible en este trabajo comenzar con una aclaración conceptual sobre la coevaluación. Posteriormente se repasan los principales beneficios que se destacan en la literatura especializada sobre esta modalidad, se presenta el estudio que se ha desarrollado durante tres años con estudiantes que comienzan sus carreras universitarias y los principales resultados obtenidos sobre la percepción y satisfacción de la experiencia realizada. 


\section{La coevaluación o evaluación compartida entre docentes y estudiantes. Una necesaria aclaración conceptual}

En la literatura pedagógica en español se utiliza el término coevaluación con significados distintos. Existen diferentes autores que han hecho referencia a esta llamativa confusión terminológica (López Pastor, 2012; Rodríguez, Ibarra y García, 2013) que se refleja en el uso polisémico de este concepto como equivalente tanto de la evaluación entre iguales como de la evaluación compartida o negociada. Actualmente estas divergencias continúan e incluso se ha llegado a recomendar el abandono definitivo del término coevaluación precisamente por considerarse que traba el entendimiento de la comunidad científica (López Pastor, 2012).

Es importante resaltar que la palabra "coevaluación" no aparece en el Diccionario de la lengua española, por lo que las interpretaciones semánticas continúan abiertas. Así, existen autores que utilizan este concepto para designar aquella evaluación que realizan unos estudiantes a otros compañeros (véanse los trabajos de Fernández March, 2010; Gessa, 2011; López Pastor, Pérez Pueyo, Barba y Lorente, 2005, 2016; Picornell, 2014;) y otros investigadores que proponen este término como la evaluación que se realiza de forma conjunta y negociada entre docentes y estudiantes y cuya responsabilidad es compartida entre ambos (véanse los trabajos de Álvarez Rojo y otros, 2011; Gómez, Rodríguez e Ibarra, 2011, 2013; Ibarra y Rodríguez, 2014; Platero, Benítez y Rodríguez, 2012; Quesada, García y Gómez, 2016). Aunque esta dicotomía es la más extendida, también se ha utilizado el término coevaluación para designar otras realidades incluso más abiertas, en este sentido Palacios (1996) la entiende como una evaluación conjunta y negociada con dos o más intervinientes, sean estos profesor-estudiante, estudianteestudiante, familia-estudiante, etc.

En este trabajo se utiliza el concepto de coevaluación como aquella evaluación que es realizada y negociada de forma conjunta entre docentes y estudiantes, en la que la responsabilidad es compartida, en la que es imprescindible el diálogo y en la que se prima llegar a un consenso sobre las valoraciones realizadas. De esta forma, consideramos que este significado del concepto de coevaluación es coherente con la noción cada vez más extendida en los estudios educativos en Educación Superior a nivel internacional, a la vez que es igualmente congruente con las nomenclaturas derivadas del inglés: coevaluación (co-assessment), evaluación entre iguales (peer assessment) y autoevaluación (self-assessment), evitando de dicho modo usos erróneos, como los que aparecen en algunos artículos traducidos del español al inglés y promoviendo la convergencia de significados para mejorar el entendimiento mutuo y los propios avances en este tópico.

\section{Delimitando el concepto de coevaluación o evaluación compartida en la universidad}

Existen multitud de expresiones en la literatura especializada en español para hacer referencia a la evaluación compartida y dialogada, no solo coevaluación, sino que también podemos encontrarla con las etiquetas de co-evaluación, evaluación 
colaborativa, evaluación cooperativa, evaluación negociada o evaluación compartida, designando todos ellos, en ocasiones con algunos matices, la misma realidad participativa.

La coevaluación (co-assessment en inglés) fue definida por Falchikov (1986) como aquella en la que estudiantes y profesorado negocian y discuten criterios de evaluación, así como la calificación final. Dochy, Segers y Sluijsmans (1999) lo conciben como el esfuerzo conjunto que realizan docentes y estudiantes para conseguir un objetivo compartido. Gómez, Rodríguez e Ibarra (2011) entienden que la coevaluación es la valoración conjunta de profesorado y estudiantes sobre el aprendizaje demostrado por este último. Por su parte, Deeley (2014) la concibe como aquella que requiere que estudiante y docente acuerden a través de la negociación y argumentación una calificación basada en evidencias.

Teniendo en consideración y poniendo en común las distintas definiciones de la coevaluación, se comparte el hecho de que tanto estudiante como docente evalúan conjuntamente el desempeño del primero. Por lo tanto, se puede deducir que la coevaluación o evaluación compartida es una modalidad de evaluación participativa y consensuada en la que profesorado y estudiante realizan una apreciación conjunta y negociada de la actuación o tarea del estudiante, si bien, como indican Bovill y Bulley (2011) puede existir una gran variedad en la calidad y cantidad de dicha colaboración.

Del mismo modo, podemos considerar la coevaluación como una modalidad participativa compuesta por una parte de autoevaluación del estudiante (que evalúa su propia tarea), y otra parte de heteroevaluación (evaluar a otro) a modo de evaluación del profesorado, dando como resultado la interacción y consenso dialógico (Quesada, García y Gómez, 2016), poniéndose además en práctica estrategias como el criticismo, la argumentación, la reflexión, el diálogo y la negociación. El hecho de que el alumnado pueda autoevaluar su actuación y que esta se pueda contrastar con la valoración del profesorado, discutir al respecto y argumentarla fomenta no solamente el aprendizaje sobre la tarea y los contenidos trabajados sino también la autorregulación del aprendizaje, al ofrecerse la oportunidad de formarse una idea más concreta de su propia actuación y de las valoraciones ofrecidas. Siguiendo esta línea, Kurt (2014) entiende la coevaluación o evaluación colaborativa directamente como una combinación de autoevaluación, evaluación entre iguales, evaluación del profesorado y una evaluación negociada.

De una u otra forma, es necesario aclarar que para que se pueda considerar que se está utilizando la modalidad de coevaluación o evaluación compartida, la evaluación ejecutada por el estudiante y la evaluación del docente se deben realizar de forma conjunta y debe llevarse a cabo un proceso de negociación y consenso, en caso contrario, realmente estaríamos considerando dos modalidades distintas por separado: la autoevaluación y la evaluación del profesorado (Quesada, García y Gómez, 2016). Esta modalidad de evaluación participativa, a la vez, ofrece la posibilidad a los estudiantes de evaluarse a sí mismos al tiempo que permite que el docente mantenga su responsabilidad en el proceso de evaluación. Si bien, para que se lleve a cabo la modalidad de coevaluación o evaluación colaborativa no se hace necesario que los estudiantes califiquen sus actuaciones, sí es necesario que participen en el proceso de clarificación de objetivos y normas, así como en el desarrollo del procedimiento de evaluación (Van der Bergh y otros, 2006), es decir, en la evaluación propiamente dicha o valoración de la actuación. 
De una forma más aglutinadora y ambiciosa para Pain, Bull y Brna (1996) la coevaluación puede aplicarse a un docente y a un estudiante que trabajan juntos para formar una comprensión mutua de los conocimientos de este último, así, el objetivo compartido es proporcionar una evaluación común acorde con los aprendizajes del estudiante, implicando igualmente que ambas partes negocien los detalles de la evaluación y las valoraciones aportadas.

\section{4. ¿Por qué utilizar la coevaluación en la universidad?}

Como se ha indicado anteriormente, la coevaluación o evaluación compartida es la modalidad participativa con los límites conceptuales menos definidos en la literatura, además de ser la que menos se utiliza en la práctica universitaria y de la que menos publicaciones y estudios se poseen. A pesar de esto, se pueden encontrar diversos e interesantes beneficios de la utilización de la coevaluación en la universidad para la mejora del aprendizaje de los estudiantes, concretamente los siguientes:

$\checkmark$ Permite mejorar las habilidades relacionadas con la función de evaluar, especialmente con la capacidad de autoevaluar el propio trabajo (Álvarez Valdivia, 2008; Deeley, 2014, Quesada, Gómez y Cubero, 2015), al ponerse en juego tanto estrategias de autoevaluación como el contraste de las propias valoraciones con las del profesorado.

$\checkmark$ El alumnado puede aumentar la comprensión del significado y repercusiones de su propio rendimiento (Deeley, 2014).

$\checkmark$ Con la coevaluación se promueve la reflexión y un aprendizaje auténtico y profundo de los procesos y productos evaluados (Knight y Yorke, 2003).

$\checkmark$ Logra mejorar la autoestima y la autoconfianza del alumnado participante (Boud y Falchikov, 2006; Knight y Yorke, 2003).

$\checkmark$ Aumenta el esfuerzo, la motivación y la implicación del alumnado (Dochy, Segers y Sluijsmans, 1999).

$\checkmark$ Con su práctica se potencian habilidades comunicativas, de negociación y argumentación (Quesada, García y Gómez, 2016).

$\checkmark$ Mejora las interacciones, la comunicación y la confianza mutua entre estudiantes y profesorado (Quesada, Gómez y Cubero, 2015), asimismo promueve la empatía entre participantes, permitiendo el cambio y la asunción de diferentes roles entre los implicados (Álvarez Valdivia, 2008).

Como se puede comprobar, la coevaluación posee importantes posibilidades formativas para optimizar el proceso de enseñanza y aprendizaje, especialmente cuando se usa de forma progresiva y conjunta con una evaluación formativa. Por ello, consideramos que la puesta en práctica de esta modalidad de evaluación puede favorecer que el alumnado tome las riendas de su propio proceso de aprendizaje, potenciando la autonomía y la autorregulación. 


\section{Objetivos}

Pickford y Brown (2006) plantean que los enfoques de evaluación originales e imaginativos tienen un impacto positivo en el compromiso y en el nivel de logro de los estudiantes. Partiendo de esta premisa, y teniendo en consideración los posibles beneficios que se derivan de la coevaluación, en este estudio se decidió implementar esta modalidad participativa de evaluación, siendo el objetivo general de la investigación el siguiente:

Analizar la potencialidad para el aprendizaje de un proceso de coevaluación desde la perspectiva de los estudiantes universitarios implicados.

A su vez, se consideraron como objetivos específicos:

$\checkmark$ Comprobar el grado de satisfacción y la percepción general del alumnado que evalúa y califica conjuntamente con el docente sus prácticas grupales.

$\checkmark$ Conocer los aspectos positivos y negativos que detectan los participantes durante una evaluación y calificación compartidas en el ámbito universitario.

\section{Método}

\subsection{Procedimiento}

La investigación se desarrolló en la asignatura "Observación sistemática y análisis de contextos", una materia obligatoria situada en el segundo semestre del primer curso del Grado en Educación Infantil impartido en la Universidad de Cádiz. En el transcurso de la misma y durante tres cursos académicos consecutivos (2012-2013, 2013-2014 y 20142015), el docente propuso a los estudiantes evaluar y calificar conjuntamente las cinco prácticas grupales -realizadas en equipos de 3 a 6 componentes- que debían elaborar durante la segunda parte de la materia. El peso de estas actividades grupales en la calificación final era del $30 \%$ y consistían en:

1. Ideas previas sobre la observación sistemática en Educación Infantil y la investigación educativa.

2. Decisiones de una observación, construcción de un sistema categorial y recogida de información en un contexto real a través de la observación sistemática.

3. Realización y grabación de una asamblea con todos los estudiantes de la clase y posterior análisis del vídeo.

4. Análisis de datos de la tarea 2 y elaboración de conclusiones.

5. Realización de una investigación sencilla mediante entrevistas y redacción de un informe.

Ya durante la presentación de la materia se explicó al alumnado la importancia de conocer y entender en profundidad no solo el desarrollo de cada una de las actividades, sino sus criterios de evaluación. Concretamente, el proceso seguido para la evaluación y calificación de cada tarea fue el siguiente:

1. Presentación de la tarea en clase. Lo primero que hacía el docente era especificar el contenido de la actividad y detallar claramente los criterios con los que 
posteriormente deberíamos evaluar conjuntamente la calidad de dicha tarea. Estas orientaciones se publicaban igualmente en el Campus Virtual de la asignatura para que en todo momento estuviera accesible para el alumnado.

2. Realización de la actividad. Los estudiantes podían trabajar grupalmente durante las sesiones prácticas presenciales para resolver las dudas y solicitar información sobre su desarrollo al docente. Posteriormente disponían de una o dos semanas para su culminación y su entrega mediante el Campus Virtual.

3. Revisión del docente. Tras la entrega de cada tarea, el docente las leía, corregía y anotaba algunos comentarios en el documento que servían de base para la retroalimentación, pero sin proponer calificación alguna.

4. Coevaluación. Durante la siguiente sesión práctica presencial, el docente se reunía con cada grupo de trabajo y dedicaba entre 10 y 15 minutos a evaluar conjuntamente y de forma dialogada y razonada la tarea realizada mediante las siguientes fases:

$\checkmark$ Lo primero en cada reunión era mostrar y explicar los comentarios realizados por el docente atendiendo a los criterios de evaluación explicitados en cada tarea.

$\checkmark$ Posteriormente se abrían las intervenciones de los estudiantes en las que mostraban su acuerdo o desacuerdo con las apreciaciones del docente, se resolvían las dudas, exponían sus percepciones y opiniones sobre las dificultades encontradas en el proceso y sobre la pertinencia del producto entregado.

$\checkmark$ Tras este diálogo entre estudiantes y docente, el profesor invitaba a los estudiantes a reflexionar y argumentar sobre la calificación que asignarían a la tarea. Se abría así un nuevo momento de diálogo, intercambio y negociación entre los estudiantes y entre estos con el docente.

$\checkmark$ El proceso concluía cuando se consensuaba una nota entre todos los participantes o bien, exclusivamente el equipo de trabajo decidía que era necesario repetir la tarea, una opción a la que siempre podían recurrir si lo consideraban pertinente. Lógicamente, si se repetía la actividad, el proceso de evaluación comenzaba de nuevo.

De forma ilustrativa, ofrecemos en la figura 1 un resumen del proceso evaluativo seguido que se acaba de describir.

Al finalizar la experiencia en cada uno de los tres cursos, se realizó un cuestionario voluntario para los estudiantes con el fin de conocer su percepción sobre el planteamiento y el desarrollo de la misma, así como para destacar aquellos beneficios, posibilidades y limitaciones de esta implantación de la práctica de la coevaluación en el ámbito universitario. 


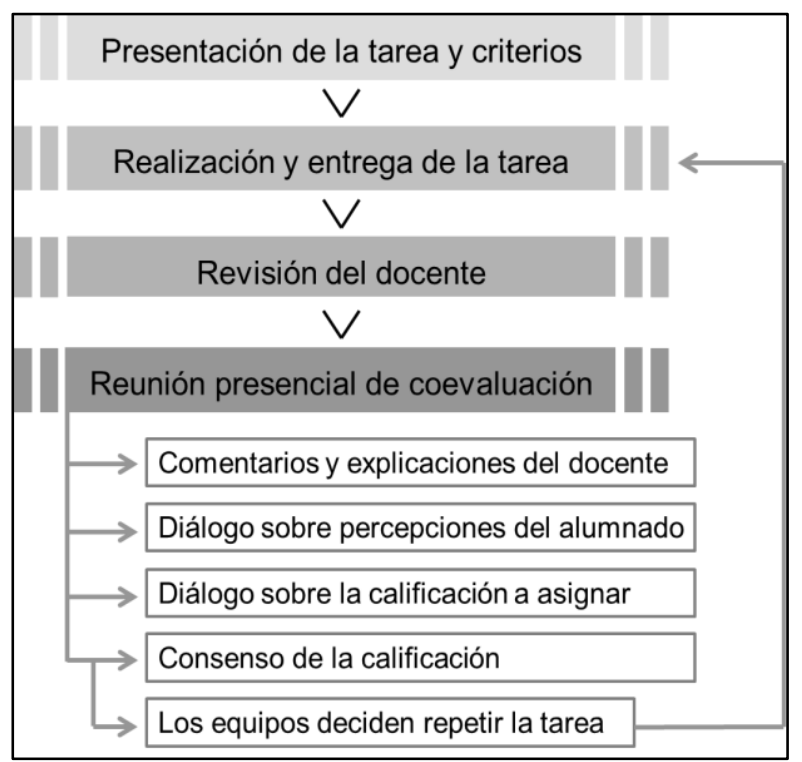

Figura 1. Procedimiento de la coevaluación Fuente: Elaboración propia.

\subsection{Participantes}

$\mathrm{Al}$ estar situada la asignatura en el primer curso de la carrera, el perfil de la mayoría de los estudiantes participantes eran jóvenes que acababan de ingresar en la universidad, bien tras superar la etapa de Bachillerato o la de Formación Profesional.

Durante los tres años en los que se desarrolló la experiencia de la coevaluación participaron un total de 192 estudiantes del Grado en Educación Infantil de la Universidad de Cádiz, de los cuales eran 178 mujeres (92,71\%) y 14 hombres (7,29\%).

Si segmentamos los participantes por cursos académicos, tal y como aparece en la tabla 1, el reparto estaría bastante equilibrado, ya que durante el curso 2012-2013 participaron 67 estudiantes (34,9\% del total), en 2013-2014 60 estudiantes $(31,25 \%)$ y durante 2014-2015 un total de 65 estudiantes (33,85\%).

Tabla 1. Participantes en la coevaluación

\begin{tabular}{lccc}
\hline \multicolumn{1}{c}{ CURSO } & MUJERES & HOMBRES & TOTAL \\
\hline $2012-2013$ & 62 & 5 & 67 \\
$2013-2014$ & 55 & 5 & 60 \\
$2014-2015$ & 61 & 4 & 65 \\
\hline Total & 178 & 14 & 192 \\
\hline Fuente: Elaboración propia & & &
\end{tabular}

Fuente: Elaboración propia.

\subsection{Instrumentación}

Para recoger las percepciones de los estudiantes implicados en la coevaluación durante los tres cursos, se diseñó un cuestionario on-line con la herramienta Google Drive ${ }^{1}$ que

${ }^{1}$ http://drive.google.com 
se enlazó desde el Campus Virtual de la asignatura. El instrumento fue diseñado de forma flexible para recoger las opiniones del alumnado, teniendo en consideración el marco de la coevaluación y siendo su contenido validado por expertos. La cumplimentación del cuestionario era totalmente voluntaria y anónima, estando compuesto por cinco preguntas que enumeramos y describimos a continuación:

1. Valoración de forma general de la experiencia en una escala de 1 a 10 y justificación argumentada de la puntuación.

2. Pregunta abierta para señalar los aspectos positivos.

3. Pregunta abierta para escribir posibles aspectos negativos.

4. Cinco afirmaciones en las que se debía seleccionar el grado en el que se estaba de acuerdo con ellas (Totalmente de acuerdo, algo de acuerdo, algo en desacuerdo y totalmente en desacuerdo). Estas afirmaciones versaban sobre los siguientes tópicos:

$\checkmark$ Preferencia de que solo el docente estableciera la calificación de las prácticas.

$\checkmark$ Percepción sobre si con la coevaluación se aprende más de los errores cometidos.

$\checkmark$ Opinión acerca de una posible sobrevaloración de las puntuaciones tras ejecutar la coevaluación.

$\checkmark$ Adecuación de la actitud y dinámica desarrollada por el docente durante su práctica.

$\checkmark$ Voluntad de utilizar la coevaluación en otras asignaturas universitarias.

5. Pregunta abierta para realizar propuestas de mejora.

\subsection{Análisis de datos}

Para el análisis de los datos se han realizado procedimientos diferenciados según la naturaleza cuantitativa o cualitativa de la información recogida.

Los datos cuantitativos procedentes de las preguntas cerradas del cuestionario ( 1 y 4 ) se han analizado mediante el cálculo de estadísticos descriptivos como medias y tablas de frecuencia con la ayuda del paquete estadístico con licencia libre "R-UCA para Windows"2.

Por su parte, los datos textuales que provienen de las preguntas abiertas del cuestionario $(1,2,3$ y 5$)$ se han analizado según el proceso general de análisis de datos cualitativos (Rodríguez, Gil y García, 1999) mediante la reducción y transformación de datos y la posterior obtención y verificación de conclusiones a través del juicio crítico entre investigadores. Las categorías y subcategorías de análisis se han establecido de forma emergente al examinar el contenido de la información, contrastándose mediante triangulación de investigadores. En el cuadro 1 se presentan las principales categorías

\footnotetext{
${ }^{2}$ http://knuth.uca.es/R/doku.php?id=instalacion_de_r_y_rcmdr:r-uca
} 
utilizadas en el análisis de las respuestas de los estudiantes participantes, las subcategorías se detallan en el próximo apartado junto con los correspondientes resultados.

Cuadro 1. Sistema de categorías para las respuestas de los participantes

\begin{tabular}{ll}
\hline \multicolumn{1}{c}{ CUESTIÓN } & \multicolumn{1}{c}{ CATEGORÍAS } \\
\hline \multirow{2}{*}{ Justificación de la valoración } & Aspectos positivos \\
& Aspectos negativos \\
\hline & Retroalimentación \\
& Colaboración y comunicación \\
Aspectos positivos & Calificación \\
& Innovación \\
& Formación \\
& Implicación \\
& Criterios \\
\hline \multirow{3}{*}{ Aspectos negativos } & Proceso \\
& Calificación \\
& Evaluación \\
& Interacción \\
Propuestas de mejora & Ninguno \\
\hline & Docente \\
& Proceso, tiempo y espacio \\
\hline
\end{tabular}

Fuente: Elaboración propia.

\section{Resultados}

Un total de 122 participantes cumplimentaron el cuestionario, lo que supone el 63,54\% de los 192 estudiantes que se implicaron en la coevaluación durante los tres años. Concretamente en 2012-2013 contestaron 41 (61,19\% de los estudiantes implicados ese curso), en 2013-2014 fueron 40 (66,67\%) y en 2015 nuevamente 41 (63,08\%).

Los resultados se comentan a continuación organizando el discurso por cada una de las preguntas del cuestionario para facilitar su exposición.

\subsection{Valoración general y justificación}

En una escala de 1 (mínimo) a 10 (máximo), la valoración general de los 122 estudiantes sobre la experiencia de la coevaluación de las prácticas grupales fue de media de 8,99 (0,95 de desviación típica). Un dato muy favorable que además se fue incrementando a lo largo de los tres cursos que duró la experiencia, tal y como se puede observar en la figura 2 sobre la tendencia de la media, siendo en 2013 de 8,78, en 2014 de 8,93 y en 2015 de 9,27. Una tendencia positiva que, aun teniendo en consideración las diferencias entre los grupos de estudiantes en cada curso, puede deberse al aumento de la experiencia del docente y de la aplicación de las sugerencias de mejora que año tras año realizaba el alumnado. 


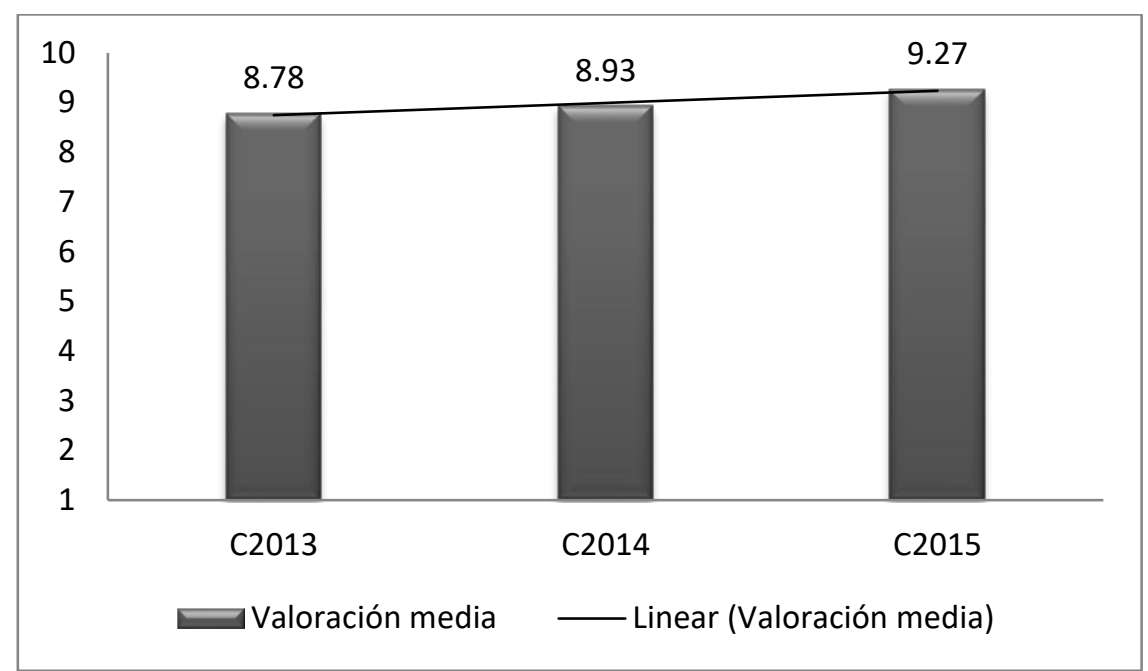

Figura 2. Tendencia de la media de las valoraciones de los estudiantes Fuente: Elaboración propia.

La frecuencia global de las 122 puntuaciones fueron las siguientes: 44 estudiantes valoraron la actividad con un $10(36,1 \%), 42$ personas con un $9(34,4 \%), 28$ participantes eligieron un 8 (23\%), 7 estudiantes la valoración $7(5,7 \%)$ y 1 la puntuación de $6(0,8 \%)$.

Parece lógico que ante unas puntuaciones tan elevadas, las justificaciones de las valoraciones por parte de los estudiantes coincidan mayormente con los aspectos positivos detectados durante la experiencia. Así, las mayores referencias las posee el tópico de la retroalimentación, ya que con esta modalidad evaluativa 81 estudiantes $(66,39 \%)$ consideraban que han sido conscientes, han reflexionado y han aprendido de los errores cometidos. Algunos ejemplos de estas contestaciones son los siguientes:

Esta forma de evaluación es muy importante ya que nosotros podemos ver los fallos y aprender de ellos. (P14-05)

Creo que es una forma buena de evaluar, ya que, entre todos podemos ver nuestros errores para asi aprender de ellos, podemos justificar algo que el docente no haya comprendido, y además, podemos llegar a un acuerdo a la hora de evaluarnos. (P15-26)

Por otra parte, 35 estudiantes (28,69\%) destacaron los beneficios de que la evaluación se haga de forma conjunta entre alumnado y docente y otros 20 (16,39\%) que es algo positivo porque se tiene en consideración la opinión de los estudiantes. Ejemplos de estas respuestas que priman la colaboración y la comunicación necesarias en la coevaluación son los siguientes:

Personalmente pienso que es una estrategia que favorece y enriquece tanto al alumno/a como al profesor, ya que ambos escuchan a las dos partes, se justifican y pueden ver los errores que han cometido para corregirlos posteriormente. (P13-14)

Me parece una muy buena idea que el docente tenga en cuenta las opiniones de los/las alumnos/as, que se tenga en cuenta el esfuerzo realizado por cada persona. (P15-35)

Completan las justificaciones de la valoración general otros aspectos positivos que detallaremos en el próximo apartado, como la posibilidad de participar en la calificación de las actividades (21), que es algo innovador para el estudiante (18) y que de esta forma se aumenta la formación en el ámbito de la evaluación (14). 
No obstante, aparecieron también algunos aspectos negativos en estas justificaciones, concretamente que esta dinámica evaluativa podría convertirse en una situación tensa e incómoda para algunos estudiantes (3 referencias), que resulta complejo evaluar el trabajo propio (2) y que los grupos de prácticas no conocen en ningún momento la nota que les daría únicamente el docente (2). Un ejemplo de este último argumento descrito fue:

Creo que de esta manera no se sabe realmente cómo la valoraría el profesor, es decir, sin saber quiénes han hecho la práctica ni la nota que ellos mismos se pondrían. Aun así, me parece una manera muy buena de evaluar. (P15-29)

\subsection{Aspectos positivos}

A continuación se ofrece el recuento de todas las referencias realizadas por el alumnado en las categorías y subcategorías de análisis como aspectos positivos tras su implicación en la coevaluación de las actividades grupales.

Tabla 2. Categorías y subcategorías de los aspectos positivos de la coevaluación

\begin{tabular}{|c|c|c|c|c|c|c|}
\hline CATEgoría & SUBCATEGORÍA & 2013 & 2014 & 2015 & TOTAL & $\begin{array}{c}\% \\
\text { TOTAL }\end{array}$ \\
\hline Retroalimentación & $\begin{array}{l}\text { Ser conscientes, reflexionar } \\
\text { y aprender de los errores } \\
\text { cometidos }\end{array}$ & 22 & 29 & 26 & 77 & 63,11 \\
\hline \multirow{2}{*}{$\begin{array}{l}\text { Colaboración y } \\
\text { comunicación }\end{array}$} & $\begin{array}{l}\text { Se hace de forma conjunta } \\
\text { profesor y grupo de } \\
\text { estudiantes }\end{array}$ & 8 & 8 & 6 & 22 & 18,03 \\
\hline & $\begin{array}{l}\text { Tener en cuenta la opinión } \\
\text { de los estudiantes }\end{array}$ & 6 & 1 & 9 & 16 & 13,11 \\
\hline \multirow[b]{2}{*}{ Formación } & Aprender a evaluar & 10 & 4 & 8 & 22 & 18,03 \\
\hline & $\begin{array}{l}\text { Desarrollar el pensamiento } \\
\text { crítico }\end{array}$ & 1 & 4 & 3 & 8 & 6,56 \\
\hline Calificación & $\begin{array}{l}\text { Participar en la calificación } \\
\text { de las actividades }\end{array}$ & 10 & 6 & 2 & 18 & 14,75 \\
\hline Criterios & $\begin{array}{l}\text { Conocer lo que el docente } \\
\text { valora }\end{array}$ & 3 & 4 & 3 & 10 & 8,20 \\
\hline \multirow{2}{*}{ Implicación } & $\begin{array}{l}\text { Se aumenta la motivación } \\
\text { del alumnado }\end{array}$ & 2 & 2 & 2 & 6 & 4,92 \\
\hline & $\begin{array}{l}\text { Mayor implicación en la } \\
\text { asignatura y la evaluación }\end{array}$ & 2 & $\mathrm{O}$ & 0 & 2 & 1,64 \\
\hline \multirow{3}{*}{ Otros } & $\begin{array}{l}\text { Se puede contrastar mejor el } \\
\text { esfuerzo realizado }\end{array}$ & 3 & $\mathrm{O}$ & 4 & 7 & 5,74 \\
\hline & Aumenta la autoestima & $\mathrm{O}$ & 1 & 1 & 2 & 1,64 \\
\hline & $\begin{array}{l}\text { Valorar el trabajo del } \\
\text { docente }\end{array}$ & 1 & $\mathrm{O}$ & $\mathrm{O}$ & 1 & 0,82 \\
\hline
\end{tabular}

Fuente: Elaboración propia.

Tal y como se refleja en la tabla 2, los estudiantes que contestaron el cuestionario consideraron nuevamente que lo mejor de esta modalidad de evaluación es que te permite ser consciente de los errores y así aprender de ellos para próximas tareas académicas e incluso profesionales. Esta importancia de la retroalimentación podemos observarla en algunas contestaciones:

Pues tener una nota justa desde mi punto de vista sobre el trabajo realizado y poder aprender de mis propios errores para no volver a cometerlos. (P 13-29) 
Esta experiencia me ha servido para ser más crítico/a con mis trabajos, exigirme más a mí mismo/a. Además he notado una gran evolución en mis trabajos, pues el conocimiento de tus fallos te ayuda a mejorar. (P15-15)

De forma similar a lo que ocurría en la justificación de las valoraciones, la categoría sobre colaboración y comunicación fue la segunda más referenciada. Después de ese tópico, se destacaron la posibilidad de aprender a evaluar y de participar en la calificación de las actividades grupales, dos temas que se pueden apreciar en las siguientes contestaciones:

El aspecto positivo sobre el aprendizaje personal de mis prácticas es que he podido realizar un juicio propio y justo sobre mi trabajo, además de aprender un poco cómo evaluar una práctica. (P13-28)

Nunca he utilizado este tipo de evaluación, pero me he dado cuenta que opinar sobre la calificación de nuestras propias prácticas ayuda más, ya que, tú mismo ves lo que realmente está bien o está mal... (P15-07)

En último lugar, los estudiantes también mencionaron que gracias a esta experiencia han podido conocer mejor los criterios con los que los docentes valoran las actividades. También consideraron que la coevaluación les permitió desarrollar el pensamiento crítico e igualmente que pudieron contrastar mejor y tener en consideración el esfuerzo que ha supuesto la realización de las distintas tareas. Veamos dos ejemplos sobre estas últimas subcategorías mencionadas:

Los aspectos positivos es que nos ponemos en el lugar del profesor .../... es difícil poner nota u opinar sobre algo que has realizado tú mismo, aunque esto nos ha ayudado a ser más críticos con nosotros a la hora de realizar algunas prácticas. (P14-13)

Este método te permite poder expresarte y te da la oportunidad de opinar ante trabajos hechos por uno mismo. De forma que el docente no solo tenga en cuenta lo que aparece reflejado en el trabajo, sino que tras la evaluación conjunta se expresen las dificultades o esfuerzos realizados, para que estos también se tengan en cuenta. (P15-16)

\subsection{Aspectos negativos}

En la tabla 3, mostrada a continuación, ofrecemos el análisis completo de las contestaciones de los participantes sobre los aspectos negativos detectados durante la experiencia de la coevaluación.

Como se puede observar, un total de 84 participantes en el cuestionario $(68,85 \%)$ consideraron que la práctica de la coevaluación no ha supuesto ni ha presentado ningún aspecto negativo mediante afirmaciones más o menos razonadas como las siguientes:

Para nada, ninguno. El trabajo en común es más beneficioso, ya que de otros compañeros y del profesor se aprende más, y cosas nuevas. (P14-37)

ro creo que para nada ha tenido un aspecto negativo, porque hemos aprendido de igual a igual y de forma más democrática. (P15-20)

De forma similar a lo que ocurría en la valoración general de la experiencia, es significativo que, tal y como se refleja en la tabla 3, durante el primer curso de aplicación se detectaron 20 de los 41 aspectos negativos totales $(48,78 \%)$ que surgieron en la investigación. En 2014 existieron 9 (21,95\%) y en 201512 (29,27\%).

El aspecto que más se referenció como negativo en un total de 10 ocasiones $(8,20 \%)$ es que la reunión presencial de coevaluación puede suponer una situación tensa o incómoda para algunos estudiantes: 
Creo que el tener que defender tu nota hace que muchas personas por vergüenza no sean cien por cien sinceros, y al final se tienda al conformismo por miedo a debatir con el professor. (P13-41)

Quizás, como es la primera vez que nos enfrentamos a esta situación la mayoría de la clase, estábamos un poco retraídos. (P15-35)

Tabla 3. Categorías y subcategorías de los aspectos negativos de la coevaluación

\begin{tabular}{|c|c|c|c|c|c|c|}
\hline CATEgoría & SUBCATEGORÍA & 2013 & 2014 & 2015 & Total & $\begin{array}{c}\% \\
\text { TotaL }\end{array}$ \\
\hline \multirow{4}{*}{ Proceso } & $\begin{array}{l}\text { Puede ser una situación tensa e } \\
\text { incómoda }\end{array}$ & 6 & 1 & 3 & 10 & 8,20 \\
\hline & $\begin{array}{l}\text { Se puede mejorar la organización } \\
\text { del tiempo }\end{array}$ & 1 & 2 & $\mathrm{O}$ & 3 & 2,46 \\
\hline & $\begin{array}{l}\text { No se da la oportunidad de } \\
\text { mejorar las prácticas a todos los } \\
\text { grupos }\end{array}$ & 1 & $\mathrm{O}$ & $\mathrm{O}$ & 1 & 0,82 \\
\hline & $\begin{array}{l}\text { No todos los componentes del } \\
\text { grupo intervienen }\end{array}$ & $\mathrm{O}$ & $\mathrm{O}$ & 1 & 1 & 0,82 \\
\hline \multirow{3}{*}{ Calificación } & $\begin{array}{l}\text { Aprovechar para calificar de } \\
\text { forma desmesurada }\end{array}$ & 6 & 2 & $\mathrm{O}$ & 8 & 6,56 \\
\hline & $\begin{array}{l}\text { Se puede infravalorar la } \\
\text { calificación }\end{array}$ & 2 & 1 & 2 & 5 & 4,10 \\
\hline & $\begin{array}{l}\text { No se sabe la nota que daría el } \\
\text { profesor }\end{array}$ & 1 & $\mathrm{O}$ & 1 & 2 & 1,64 \\
\hline Evaluación & $\begin{array}{l}\text { Es complejo evaluar el trabajo } \\
\text { propio }\end{array}$ & 3 & 3 & 3 & 9 & 7,38 \\
\hline Interacción & Trabajar en grupo & $\mathrm{O}$ & $\mathrm{O}$ & 2 & 2 & 1,64 \\
\hline Ninguno & Ninguno & 23 & 31 & 30 & 84 & 68,85 \\
\hline
\end{tabular}

Fuente: Elaboración propia.

El alumnado participante también hizo mención a las dificultades que ha encontrado al evaluar sus propios desempeños, es decir, en el componente de autoevaluación que consustancialmente posee la modalidad de la evaluación compartida:

Yo creo que no tiene ningún aspecto negativo, lo único es que la persona que corrige su propio trabajo no es totalmente objetivo con él, ya que a pesar de que puedan haber fallado, siempre van a considerar el trabajo que han tenido que hacer para realizar cada práctica. (P15-12)

Por último, resulta interesante que los otros dos aspectos negativos que más aparecen sean contradictorios entre sí, ya que hay 8 personas $(6,56 \%)$ que consideran que de esta forma se puede aprovechar la coyuntura para calificarse de forma desmesurada, mientras que otras $5(4,10 \%)$ opinan todo lo contrario, que mediante la coevaluación se puede infravalorar la calificación. Exponemos a continuación un ejemplo de cada subcategoría:

Sí, porque pienso que hay grupos que se pueden sobrevalorar las prácticas. (P13-03)

Como inconveniente, cabe decir que podemos infravalorar nuestras notas y obtener menos. (P14-32)

\subsection{Grado de acuerdo con las afirmaciones propuestas}

En la cuarta pregunta del cuestionario se les consultaba directamente a los estudiantes sobre varios aspectos clave del desarrollo de esta modalidad conjunta de evaluación entre estudiantes y docente. El alumnado debía seleccionar si estaba totalmente en desacuerdo (codificada como 1), algo en desacuerdo (2), algo de acuerdo (3) o totalmente de acuerdo (4) con ellas. Las afirmaciones propuestas fueron las siguientes: 


\section{Personalmente hubiera preferido que solo el docente estableciera la calificación sin preguntarnos}

Ante esta afirmación, el $75,41 \%$ del alumnado que contestó el cuestionario durante los tres cursos (92 personas) estaban totalmente en desacuerdo. Únicamente 7 estudiantes $(5,74 \%)$ se posicionaron algo de acuerdo (6) o totalmente de acuerdo (1) en que el docente hubiera calificado en solitario las prácticas grupales. De esta forma, la media general de las respuestas se sitúa en 1,31 con una desviación típica de 0,6.

2. Creo que de esta forma he aprendido más de los errores y tengo más claro cómo debo hacer las cosas la próxima vez

Al ser preguntados por la importancia de la retroalimentación durante la coevaluación, y en coherencia con las anteriores respuestas, 113 estudiantes $(92,62 \%)$ están totalmente de acuerdo con esta afirmación. Únicamente 4 respuestas (3,28\%) aparecen como algo (2) o totalmente en desacuerdo (2). La media global de las contestaciones a este tópico es de 3,88 con una desviación típica de 0,49 .

3. Creo sinceramente que las notas a las prácticas no han sido ajustadas, sino que se han sobrevalorado al tener la opinión de mi grupo en cuenta

Este es un aspecto que siempre se tiene presente en el desarrollo de las evaluaciones compartidas entre estudiantes y docente y del que teníamos especial interés en conocer la percepción sincera del alumnado participante.

Aunque ciertamente encontramos algo más de equilibrio que en las dos afirmaciones anteriores, 95 personas $(77,87 \%)$ se encuentran algo (39) o totalmente (56) en desacuerdo. No obstante, no es desdeñable que el 18,03\% esté algo de acuerdo (22 estudiantes) y el 4,1\% (5) totalmente de acuerdo. En esta ocasión la media general es de 1,80 con una desviación típica de 0,88 .

4. Pienso que la actitud y la dinámica desarrollada por el profesor en la evaluación de las prácticas han sido correctas

Hasta 98 personas (80,33\%) están totalmente de acuerdo en que la actitud del docente y el proceso realizado han sido pertinentes. 2 estudiantes $(1,64 \%)$ se posicionaron totalmente en desacuerdo y otros $3(2,46 \%)$ se mostraron algo en desacuerdo. La media en esta afirmación es de 3,75 con una desviación típica de 0,58. En esta ocasión es interesante observar que la media aumenta de 2013 con 3,54 hasta el 3,85 en los años 2014 y 2015, algo que viene a apoyar los resultados anteriormente comentados de un aumento progresivo en la consideración del proceso de la coevaluación.

5. Me gustaría que me pidieran mi opinión de esta forma todos los docentes de todas las asignaturas

Ante la posibilidad de extrapolar esta modalidad de evaluación a otras asignaturas universitarias, las respuestas parecen situarse abrumadoramente a favor, con 95 estudiantes $(77,87 \%)$ totalmente de acuerdo y otros 23 (18,85\%) algo de acuerdo. Solo 4 personas $(3,28 \%)$ se muestran algo (3) o totalmente (1) reacias a que se produjera esta posibilidad. La media global de este ítem es de 3,74 con una desviación típica de 0,54. 
Para finalizar esta exposición, añadimos la figura 3 donde se puede observar la frecuencia y tendencia de las valoraciones medias durante los tres cursos en los que se implantó la experiencia para cada una de las afirmaciones descritas.

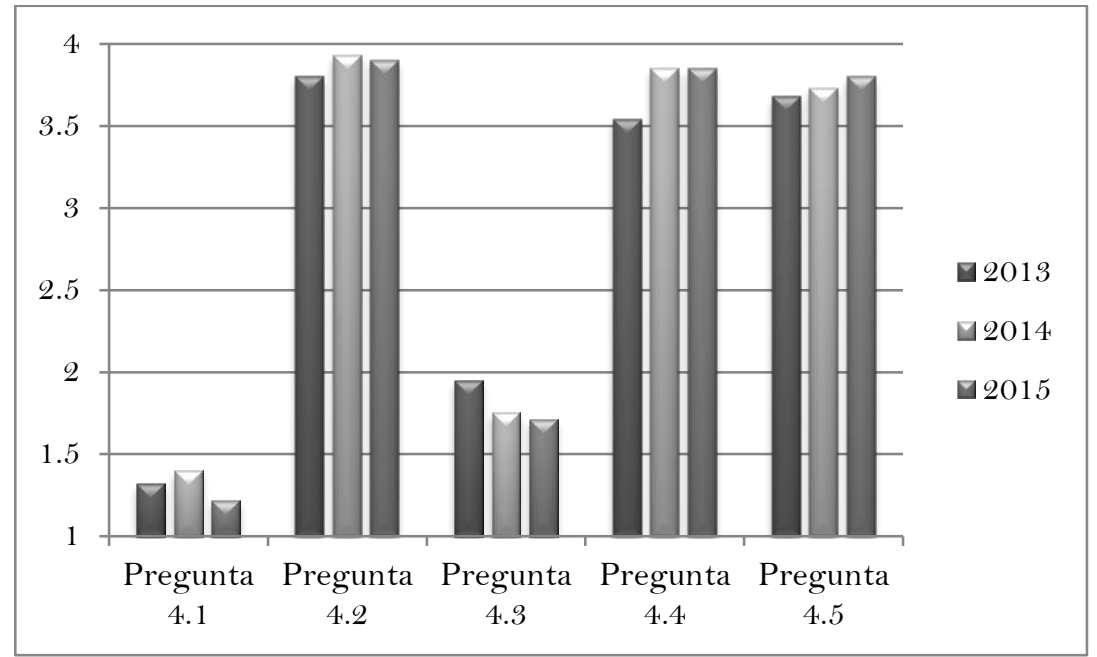

Figura 3. Frecuencia de las medias de las valoraciones a las afirmaciones sobre la coevaluación

Fuente: Elaboración propia.

\subsection{Propuestas de mejora}

Han sido múltiples y muy diversas las propuestas realizadas por los 122 estudiantes que cumplimentaron el cuestionario durante la recogida de la información. Sin embargo, solo hay dos de ellas que alcanzan las diez referencias, concretamente que el docente organizara mejor el tiempo de las correcciones (13) y que el docente expresara la nota que le pondría a las tareas grupales si fuera el único responsable de la calificación (10). Ejemplos de estas propuestas la podemos encontrar en las siguientes opiniones:

Repartiendo mejor el tiempo en las clases ya que a muchos grupos no les daba tiempo y tenían que esperar más. Una forma podría ser repartiendo todas las prácticas para que los grupos las vayan mirando y luego el docente pasa por las mesas y aunque no dé tiempo, al menos todos han visto las prácticas. (P14-20)

Pienso que lo mejor sería que el profesor nos dijera cuál considera él que es la nota adecuada y a partir de ahí entonces debatirla, no fijarnos nosotros la nota. (P15-18)

De manera menos recurrente, también encontramos otras muchas propuestas englobadas en las categorías de "Docente", "Proceso, tiempo y espacio" y "Estudiantes y grupos de trabajo" que enumeramos de forma descendente a continuación:

$\checkmark$ Que el docente enseñara ejemplos modélicos (4)

$\checkmark$ Que el docente explicara más detalladamente los criterios y cómo ha evaluado (3)

$\checkmark$ Que se tuviera que llegar a consenso entre los componentes del grupo (3)

$\checkmark$ Que los estudiantes se pusieran nota individualmente (3)

$\checkmark$ Que se realizaran las evaluaciones con todo el grupo de clase (3)

$\checkmark$ Que el docente advirtiera al grupo si se sobrevalora en su calificación (2) 
Que el docente precisara más los aspectos a mejorar (2)

Que se fomentara la confianza para participar (2)

$\checkmark$ Que los grupos explicaran primero su trabajo (2)

\section{Discusión y conclusiones}

En este estudio se ha presentado una experiencia de coevaluación o evaluación compartida realizada con estudiantes de primer curso del Grado en Educación Infantil de la Universidad de Cádiz durante tres años académicos. El objetivo general propuesto era analizar la potencialidad para el aprendizaje de un proceso de coevaluación desde la perspectiva de los estudiantes, comprobando su grado de satisfacción y valorando los aspectos positivos y negativos que detectan.

Respecto a la satisfacción del alumnado con la experiencia, las elevadas puntuaciones y su tendencia ascendente durante los tres años no solo evidencian que el alumnado apreció los elementos beneficiosos de esta modalidad evaluativa, sino que el docente pudo ir mejorando su ejecución con el tiempo, de hecho, como señalan algunos autores (Quesada, Rodríguez e Ibarra, en prensa) para que las prácticas innovadoras tengan unos efectos deseables es necesario que sean sostenibles y continuas. Con el paso de los cursos se intentó optimizar el tiempo dedicado a cada grupo, se explicaron más detalladamente el proceso y los criterios de evaluación, así como se intentó mejorar el ambiente todo lo posible en las reuniones de coevaluación para evitar situaciones incómodas para los estudiantes.

Es igualmente destacable que en la pregunta sobre los aspectos positivos de la experiencia han aparecido diversos beneficios señalados en la literatura específica. Sin duda, los estudiantes apuntan a que la mayor potencialidad de la coevaluación para el aprendizaje proviene de la valía de la retroalimentación que se ofrece y la posibilidad de ser más conscientes, reflexionar y aprender de los errores cometidos, lo cual sugiere que a través de la evaluación colaborativa se puede alcanzar un aprendizaje profundo, a la vez que favorece la autorregulación del aprendizaje para que el propio alumnado identifique sus necesidades formativas, sus metas, realice un seguimiento de lo aprendido, modifique estrategias cuando sea necesario y juzgue la calidad de sus trabajos teniendo en consideración los elementos contextuales (Boud y Falkinov, 2006). De esta forma, se puede considerar que la implicación activa del alumnado en el proceso de evaluación mejora y hace más significativa la retroalimentación aportada, ya que se sabe que el hecho de que el docente ofrezca a los estudiantes información unidireccional sobre qué y cómo deben revisar un trabajo no es una garantía de que actúen siguiendo esas orientaciones (Hounsell, 2007).

Otro beneficio relevante apuntado por parte los estudiantes ha sido considerar que a través de la coevaluación han aprendido a evaluar. Este aspecto, destacado también en diversos trabajos (Álvarez Valdivia, 2008; Deeley, 2014; Quesada, García y Gómez, 2016), se ha reflejado en un conocimiento más profundo de los criterios de evaluación, en mejorar el contraste del esfuerzo realizado, en el desarrollo de un pensamiento crítico o en la comprensión de su propia actuación o rendimiento. 
Del mismo modo, los estudiantes participantes han considerado la colaboración y la comunicación necesaria en el proceso como un elemento muy positivo, resaltando el hecho de que la evaluación se realice de forma conjunta entre estudiantes y docente, así como el hecho de tener en consideración su opinión. Esta percepción es compartida por docentes implicados en experiencias similares de coevaluación al afirmar que mediante esta práctica se había mejorado la comunicación, la confianza y el entendimiento con el alumnado, y que había existido una mayor implicación y compromiso de los estudiantes en las tareas realizadas (Quesada, Gómez y Cubero, 2015). No obstante, es necesario señalar que en esta investigación únicamente ocho estudiantes han afirmado explícitamente que les había supuesto una mayor implicación y motivación.

En efecto, no hay que olvidar que durante la evaluación colaborativa pueden darse situaciones no esperadas, así como actuaciones y efectos no deseados que influyan negativamente en el alumnado. En esta ocasión, algunos estudiantes han percibido que las reuniones dialógicas pueden convertirse en una situación tensa e incómoda, por lo que al realizar esta práctica es necesario crear un ambiente tranquilo, distendido y de confianza para que los estudiantes puedan sentirse cómodos y libres (Carless, 2012; Quesada, Rodríguez e Ibarra, 2016).

En la misma línea, algunos participantes han considerado que su implicación en la calificación podría suponer cierta desnaturalización del proceso, desvirtuándose la coevaluación mediante la sobrevaloración o infravaloración de dichas calificaciones. En este caso se debe aclarar que, en primer lugar y a pesar de la existencia de autores que sostienen realizar experiencias de calificación dialogada únicamente al final de las asignaturas (López Pastor, 2012), en esta implantación se decidió integrar desde su comienzo como una forma de involucrar de forma auténtica y significativa a los estudiantes en su proceso de evaluación universitaria. En segundo lugar, no se debe olvidar que, de una u otra forma, los aspectos más relevantes son el diálogo, la argumentación y las evidencias que fundamenten la toma de decisiones conjuntas, así como ser conscientes de la influencia de la autopercepción de cada persona o grupo que puede inducir a valoraciones demasiado laxas o estrictas.

Otro aspecto negativo identificado ha sido la complejidad manifiesta que supone evaluar el propio trabajo para el alumnado. En este sentido son diversos los estudios que hacen referencia a este ámbito, resaltando en algunos de ellos la importancia de educar progresivamente a los estudiantes en la evaluación, así como la importancia que tiene fomentar la autoevaluación para lograr la autonomía en el estudiante.

En último lugar, la organización del tiempo fue otro aspecto a mejorar que se destacó en esta investigación. El tema de la disposición temporal es un constante problema que tiene el profesorado para profundizar en metodologías o prácticas innovadoras. Dejando de lado posibles aspectos estructurales e institucionales poco proclives, será necesario en este aspecto mejorar -dentro de las posibilidades- la organización del tiempo y asegurarse de que se dispone de suficiente margen para poder realizar la coevaluación considerándola desde el inicio de la planificación docente y teniendo en cuenta que se tiende a subestimar el tiempo necesario para realizar cada una de las tareas (Quesada, Rodríguez, Ibarra, en prensa), asumiendo además una posición crítica y reflexiva ante la propia práctica docente que disminuya las divergencias entre docentes y estudiantes 
sobre los sistemas de evaluación de aprendizajes en la universidad (Hamodi Galán, López Pastor y López Pastor, 2015).

Las preferencias del alumnado parecen claras. El 94,26\% de los participantes en esta experiencia ha preferido establecer las valoraciones de forma conjunta antes de que lo realizara el docente en solitario, un porcentaje idéntico (94\%) al hallado en otra investigación relacionada con la evaluación colaborativa en entornos de e-Learning en la que igualmente los estudiantes involucrados creían que el tutor no debía evaluar en solitario sus trabajos (McConnell, 2002).

A pesar de los resultados obtenidos, no se deben obviar algunas limitaciones del presente estudio, realizado en una sola materia de una única titulación, con un solo docente como responsable y utilizando exclusivamente el cuestionario on-line como instrumento para la recogida de información. Por ello, se hace imprescindible continuar la investigación sobre este tópico utilizando mayor diversidad metodológica y de participantes, integrando y comparando las percepciones de los estudiantes con las de docentes, analizando la posible influencia en las calificaciones de la utilización de esta modalidad evaluativa o abordando las posibilidades y limitaciones que aportan las tecnologías como soporte de la coevaluación en el ámbito universitario.

Para concluir debemos resaltar la importancia manifiesta que tiene la actitud del docente en la puesta en práctica de experiencias de coevaluación. Si se desea tener posibilidades de éxito será necesario que exista un diálogo verdadero entre los protagonistas, que se establezca un marco de confianza y que exista sensibilidad y flexibilidad a cada realidad individual y a cada forma de expresión. Solo así se podrá fomentar con la coevaluación una evaluación inclusiva, democrática, crítica, optimista y justa que permita convertirla en una posible estrategia para lograr una evaluación para la Justicia Social (Murillo e Hidalgo, 2015).

\section{Referencias}

Álvarez Rojo, V., Padilla Carmona, M. T., Rodríguez Santero, J., Torres Gordillo, J. J. y Suárez Ortega, M. (2011). Análisis de la participación del alumnado universitario en la evaluación de su aprendizaje. Revista Española de Pedagogía, 250, 401-426.

Álvarez Valdivia, I. (2008). La coevaluación como alternativa para mejorar la calidad del aprendizaje de los estudiantes universitarios: Valoración de una experiencia. Revista Interuniversitaria de Formación del Profesorado, 22(3), 127-140.

Boud, D. y Falchikov, N. (2006). Aligning assessment with long-term learning. Assessment and Evaluation in Higher Education, 31(4), 399-413. https://doi.org/10.1080/02602930600679050

Bovill, C. y Bulley, C. J. (2011). A model of active student participation in curriculum design: Exploring desirability and possibility. En C. Rust (Ed.), Improving student learning global theories and local practices: Institutional, disciplinary and cultural variations (pp. 176-88). Oxford: The Oxford Centre for Staff and Educational Development.

Carless, D. (2007). Learning-oriented assessment: Conceptual bases and practical implications. Innovations in Education and Teaching International, 44(1), 57-66. https://doi.org/10.1080/14703290601081332 
Carless, D. (2012). Trust and its role in facilitating dialogic feedback. En D. Boud y L. Molloy (Eds.), Feedback in higher and professional education (pp. 90-103). Londres: Routledge.

Deeley, S. (2014). Summative co-assessment: A deep learning approach to enhancing employability skills and attributes. Active Learning in Higher Education, 15(1) 39-51. https://doi.org/10.1177/1469787413514649

Dochy, F., Segers M. y Sluijsmans, D. (1999). The use of self-, peer and co-assessment in higher education: A review. Studies in Higher Education, 24(3), 331-350. https://doi.org/10.1080/03075079912331379935

Falchikov, N. (1986). Product comparisons and process benefits of collaborative peer group and self-assessments. Assessment \& Evaluation in Higher Education, 11(2), 144-166. https://doi.org/10.1080/0260293860110206

Fernández, A. (2010). La evaluación orientada al aprendizaje en un modelo de formación por competencias en la educación universitaria. Revista de Docencia Universitaria, 8(1), 11-34.

Gessa Perera, A. (2011). La coevaluación como metodología complementaria de la evaluación del aprendizaje. Análisis y reflexión en las aulas universitarias. Revista de Educación, 354, 749764.

Gómez Ruiz, M. A., Rodríguez Gómez, G. e Ibarra Sáiz, M. S. (2011). Caracterización de la eevaluación orientada al e-aprendizaje. En G. Rodríguez y M. S. Ibarra (Eds.), e-Evaluación orientada al e-aprendizaje estratégico en educación superior (pp. 33-56). Madrid: Narcea.

Gómez Ruiz, M. A., Rodríguez Gómez, G. e Ibarra Sáiz, M. S. (2013). COMPES: Autoinforme sobre las competencias básicas relacionadas con la evaluación de los estudiantes universitarios. Estudios sobre Educación, 24, 197-224.

Hamodi Galán, C., López Pastor, A. T. y López Pastor, V. L. (2015). Percepciones de alumnos, egresados y profesores sobre los sistemas de evaluación del aprendizaje. @ tic. Revista d'Innovació Educativa, 14, 71-81. https://doi.org/10.7203/attic.14.4175.

Hounsell, D. (2007). Towards more sustainable feedback to students. En D. Boud y N. Falchikov (Eds.), Rethinking assessment in higher education. Learning for the longer term (pp. 101-113). Londres: Routledge.

Ibarra Saiz, M. S. y Rodríguez Gómez, G. (2014). Modalidades participativas de evaluación: Un análisis de la percepción del profesorado y de los estudiantes universitarios. Revista de Investigación Educativa, 32(2), 339-361.

Knight, P. T. y Yorke, M. (2003). Assessment, learning and employability. Maidenhead: SRHE/Open University Press/McGraw-Hill Education.

Kurt, M. (2014). Collaborative Assessment: Fostering ownership in assessment. Education, 134(3), 332-339.

López Pastor, V. M. (2012). Evaluación formativa y compartida en la universidad: Clarificación de conceptos y propuestas de intervención desde la Red Interuniversitaria de Evaluación Formativa. Psychology, Society \& Education, 4(1), 117-130.

López Pastor, V. M., González Pascual, M. y Barba Martín, J. J. (2005). La participación del alumnado en la evaluación. La autoevaluación, la coevaluación y la evaluación compartida. Tándem: Didáctica de la Educación Física, 17, 21-37

López Pastor, V. M., Pérez Pueyo, A., Barba, J. J. y Lorente Catalán, E. (2016). Percepción del alumnado sobre la utilización de una escala graduada para la autoevaluación y la 
coevaluación de trabajos escritos en la formación inicial del profesorado de educación física (FIPEF). Cultura, Ciencia, Deporte, 11 (31), 37-50. https://doi.org/10.12800/ccd.v11i31.641

McConnell, D. (2002). The experience of collaborative assessment in e-learning. Studies in Continuing Education, 24(1), 73-92. https://doi.org/10.1080/01580370220130459

Murillo, F. J. e Hidalgo, N. (2015). Enfoques fundamentantes de la Evaluación de Estudiantes para la Justicia Social. Revista Iberoamericana de Evaluación Educativa, 8(1), 43-61.

Pain, H., Bull, S. y Brna, P. (1996). A student model 'for its own sake'. Recuperado de http://homepages.inf.ed.ac.uk/pbrna/papers/euroaiedpapers96/smpaper/smpaper.html

Palacios Árbol, S. G. (1998). Marco referencial para la evaluación de un proyecto educativo. Educación XX1, 1(1), 93-128. https://doi.org/10.5944/educxx 1.1.1.399

Pickford, R. y Brown, S. (2006). Assessing skills and practice. Londres: Routledge.

Picornell Lucas, A. (2014). La coevaluación de competencias en el Grado en Trabajo Social mediante el uso de la rúbrica. Revista Internacional de Trabajo Social y Bienestar, 3, 17-14.

Platero Jaiome, M., Benito Hernández, S. y Rodríguez Duarte, A. (2012). Co-evaluación y asignación de roles, una experiencia de innovación docente universitaria. Docencia e Investigación, 22, 7-29.

Quesada, V., García Jiménez, E. y Gómez Ruiz, M. A. (2016). Student participation in assessment processes: A way forward. En E. Cano y G. Ion (Eds.), Innovative practices for higher education assessment and measurement (pp. 228-249). Hershey, PE: IGI Global.

Quesada Serra, V., Gómez Ruiz, M. A. y Cubero Ibáñez, J. (2015). La evaluación colaborativa en Educación Superior: Descripción de una experiencia con alumnos de primer curso. Universitat de Girona (Ed.), V Congreso Internacional UNIVEST'15. Los retos de mejorar la evaluación (pp. 562-566). Girona: Institut de Ciències de l’Educació Josep Pallach.

Quesada Serra, V., Rodríguez Gómez, G. e Ibarra Sáiz, M. S. (en prensa). La planificación y la innovación de la evaluación en Educación Superior: El profesorado y su perspectiva. Revista de Investigación Educativa.

Quesada Serra, V., Rodríguez Gómez, G. e Ibarra Sáiz, M. S. (2016). What are we missing? Spanish lecturers' perceptions of their assessment practices. Innovations in Education and Teaching International, 53(1), 48-59. doi:10.1080/14703297.2014.930353

Rodríguez Gómez G., Gil Flores, J. y García Jiménez, E. (1999). Metodología de la investigación educativa. Archidona: Aljibe.

Rodríguez Gómez, G., Ibarra Sáiz, M. S., Gallego Noche, B., Gómez Ruiz, M. A. y Quesada Serra, V. (2012). La voz del estudiante en la evaluación del aprendizaje: Un camino por recorrer en la universidad. RELIEVE, $18(2)$, 1-21. https://doi.org/10.7203/relieve.18.2.1985.

Rodríguez Gómez, G., Ibarra Sáiz, M. S. y García Jiménez, E. (2013). Autoevaluación, evaluación entre iguales y coevaluación: Conceptualización y práctica en las universidades españolas. Revista de Investigación en Educación, 1 1(2), 198-210.

Rodríguez Gomez, G., Quesada Serra, V. e Ibarra Sáiz, M. S. (2016). Learning-oriented eassessment: The effects of a training and guidance programme on lecturers' perceptions. Assessment $\Xi^{2}$ Evaluation in Higher Education, 31 (1), 35-52. https://doi.org/10.1080/02602938.2014.979132

Van der Bergh, V., Mortelmans, D., Spooren, P., Van Petegem, P., Gijbels, D. y Vanthournout, G. (2006). New assessment modes within project-based education - The stakeholders. 
Studies in Educational Evaluation, 32(4), 345-368.

https://doi.org/10.1016/j.stueduc.2006.10.005

\section{Breve CV de los autores}

\section{Miguel Ángel Gómez Ruiz}

Es Maestro, Licenciado en Pedagogía y Doctor en Posgrado Oficial de Psicología, Educación y Desarrollo. Actualmente es Profesor Ayudante Doctor en el Área de Didáctica y Organización Escolar del Departamento de Didáctica de la Universidad de Cádiz. Pertenece al Grupo de Investigación EVALfor (Evaluación en Contextos Formativos) y ha participado en diversos proyectos a nivel autonómico, nacional, europeo y de cooperación internacional sobre evaluación de competencias y aprendizajes en Educación Superior. Sus líneas de investigación son la Evaluación orientada al aprendizaje y participación en procesos de evaluación, así como las nuevas tecnologías aplicadas a la educación, aplicaciones innovadoras y desarrollo de material multimedia, habiendo realizado diversas publicaciones sobre estas temáticas. ORCID ID: 0000-00032418-9525. Email: miguel.gomez@uca.es

\section{Victoria Quesada Serra}

Es Licenciada en Pedagogía, Máster en Orientación y Evaluación Socioeducativa y Doctora en Ciencias Sociales y Jurídicas. Actualmente es Profesora en el Área de Métodos de Investigación y Diagnóstico en Educación del Departamento de Didáctica de la Universidad de Cádiz y miembro activo del Grupo de Investigación EVALfor (Evaluación en Contextos Formativos). Ha participado en varios proyectos y congresos a nivel nacional e internacional. Sus líneas de investigación preferentes son la evaluación para el aprendizaje en educación superior, la participación de los estudiantes en la evaluación, la formación en evaluación y el desarrollo de la competencia evaluadora en docentes y estudiantes. ORCID ID: 0000-0002-8881-2358. Email: victoria.quesada@uca.es 